\title{
A rare case of abdominal pregnancy: diagnostic and therapeutic challenges
}

\author{
Nishita A. Mehta*, Michelle N. Fonseca
}

Department of Obstetrics and Gynecology, Lokmanya Tilak Municipal Medical College and General Hospital, Sion, Mumbai, Maharashtra, India

Received: 27 December 2019

Revised: 24 January 2020

Accepted: 29 January 2020

\section{*Correspondence:}

Dr. Nishita A. Mehta,

E-mail: nishimehta103@gmail.com

Copyright: () the author(s), publisher and licensee Medip Academy. This is an open-access article distributed under the terms of the Creative Commons Attribution Non-Commercial License, which permits unrestricted non-commercial use, distribution, and reproduction in any medium, provided the original work is properly cited.

\begin{abstract}
Abdominal pregnancy refers to a pregnancy that has implanted in the abdominal cavity, the estimated incidence being 1 per 30,000 births. A 36-year-old primigravida with term pregnancy with fetal demise was referred to us. Examination was suggestive of single foetus of 30 weeks' gestation with longitudinal lie and cephalic presentation with absent foetal heart sounds. The cervical os was closed, uneffaced. Ultrasound done at 18 weeks' gestation had reported pregnancy in a bicornuate uterus. Present ultrasound revealed intrauterine foetal demise of 28.4 weeks. Cervical ripening, done using prostaglandins, mechanical dilation with Foley's catheter and oxytocin, had failed, and thus patient was taken up for surgery. Findings revealed an abdominal pregnancy with a macerated fetus of 1070 grams (severely growth restricted). Placenta was found to be implanted on multiple areas of both small and large intestine and posterior peritoneum. Placenta was left in situ. Postoperative recovery was uneventful. She was given higher antibiotics, 4 doses of tablet mifepristone $200 \mathrm{mg}$ and monitored regularly with ultrasound/ MRI and bHCG which showed slow placental resorption. Conclusion- Abdominal pregnancies, associated with a high maternal and perinatal morbidity and mortality, are diagnosed preoperatively only in $45 \%$ of cases. Thus, a high index of suspicion and improvement in diagnosis is the need of the hour. Successful management includes prompt intraoperative recognition and management of the placenta (we advocate leaving the placenta in situ), multidisciplinary approach with involvement of surgeons and interventional radiologists, access to blood products, meticulous postoperative care and close observation during the subsequent delayed reabsorption.
\end{abstract}

Keywords: Abdominal pregnancy, Intrauterine foetal death, Laparotomy, Mifepristone, Ectopic pregnancy

\section{INTRODUCTION}

Abdominal pregnancy refers to a pregnancy that has implanted in the abdominal or peritoneal cavity external to the uterine cavity, fallopian tubes or ovaries. The estimated incidence is 1 in 10,000 to 1 in 30,000 births and 1.4 percent of ectopic pregnancies. ${ }^{1}$ Extrauterine abdominal pregnancy rarely progresses to near term, and in the few cases reported, diagnosis has frequently been missed during antenatal care. Authors report a similar case of a primigravida with 9 months' gestation with foetal death, where abdominal pregnancy was diagnosed intraoperatively, and managed conservatively with retaining the placenta in situ.

\section{CASE REPORT}

A 36-year-old primigravida married since 16 years, with spontaneous conception with 37 weeks' gestation was referred to our obstetrics emergency department for failure of induction of labour, done for an intrauterine foetal death. Prior to referral to this centre, she had been 
induced with dinoprostone gel, $0.5 \mathrm{mg}$ inserted twice at a 6-hourly interval, Foley's catheter, tablet misoprostol inserted at 4 hourly intervals up to a total of 200 micrograms and oxytocin. She had not been regular with her antenatal check-ups, thus intrauterine growth restriction had been missed, but had an otherwise uneventful antenatal history. An ultrasound of 18 weeks was suggestive of pregnancy in a bicornuate uterus.

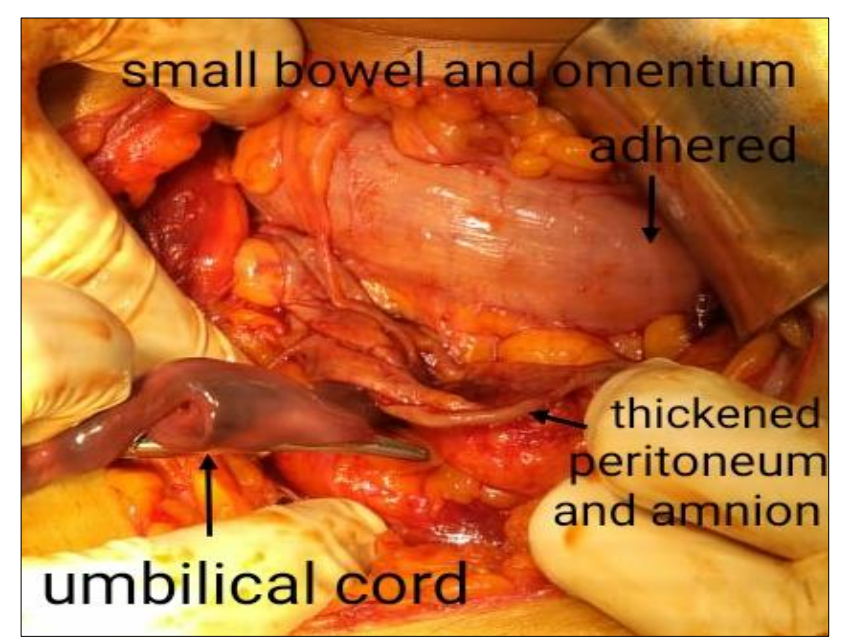

Figure 1: Intraoperative findings after delivery of foetus- thickened layer of peritoneum around the amniotic sac, with bowel and omentum adherent to it.

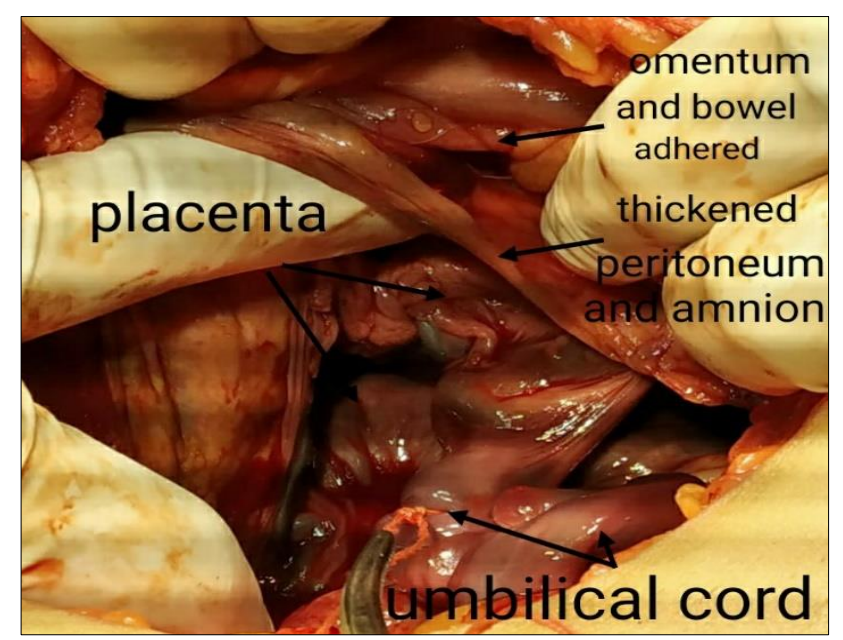

Figure 2: Intraoperative findings- placenta seen adhered to bowel, omentum and posterior peritoneum.

She had no other significant obstetric, medical or surgical history. On examination, her vital parameters were normal. Per abdomen examination revealed a uterus of 30 weeks' gestation with single foetus in longitudinal lie and cephalic presentation with absent foetal heart sounds. On vaginal examination, the cervical os was closed, uneffaced. Blood investigations were normal, and ultrasound revealed intrauterine foetal demise of 28.4 weeks' gestation.

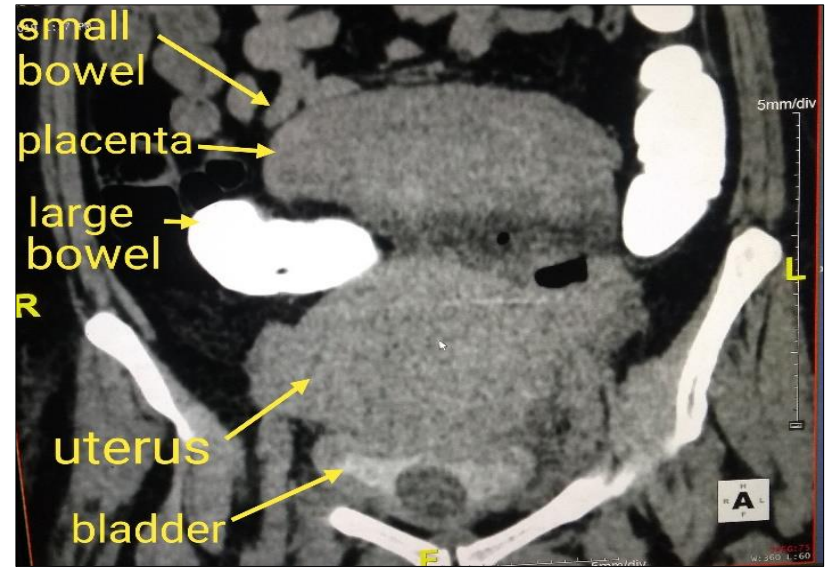

Figure 3: Postoperative CT scan- placenta seen situated outside the uterus, adherent to small and large bowel.

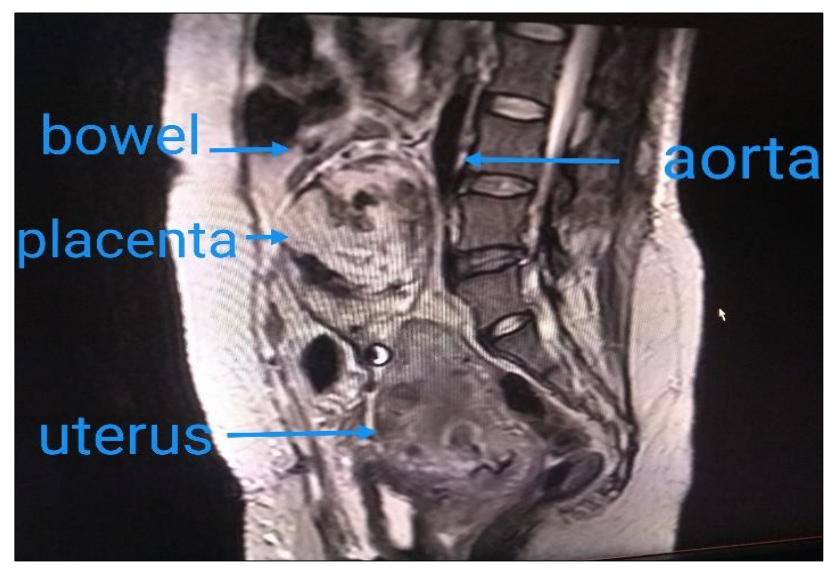

Figure 4: MRI done postoperatively depicting the placenta in the abdominal cavity.

She was taken up for caesarean section in view of failure of induction of labour. Intraoperative findings revealed a thickened layer of peritoneum around the amniotic sac, with bowel loops stretched out and adherent to it, which was evident due to appendices epiploicae and omentum seen adhered. A transverse incision was taken on a transparent portion of the lower part of the amniotic sac. A male macerated foetus of 1070 grams was delivered. Placenta was found to be implanted on and adherent to multiple areas on both small and large intestine, omentum and posterior peritoneum. (Figure 1,2) The umbilical cord was clamped, cut and ligated near the placental attachment and decision to keep placenta in situ was taken. After delivery of the foetus, the uterus was seen, which was deep in the pelvis and posterior to the amniotic sac, hence obscured until delivery. It was normal in shape and 6-8 weeks' size, and bilateral adnexa appeared normal. Hemostasis was achieved and abdomen closed. Intraoperative blood loss was only $500 \mathrm{ml}$. Postoperatively, the patient was monitored meticulously. She was given 4 doses of tablet mifepristone $200 \mathrm{mg}$ weekly. She had 2 fever spikes 5 days post operatively and was admitted to the ICU for treatment of pneumonia, 
for which she was treated symptomatically and antibiotics stepped up. Weekly MRI/CT scan were done to monitor the placental size, and beta human chorionic gonadotrophin tested on days 7, 14 and 21 was 984, 260 and $5.77 \mathrm{mIU} / \mathrm{ml}$ respectively (Figure 3).

Her laboratory and vital parameters remained normal. MRI reported a $13 \times 8 \times 6 \mathrm{~cm}$ solid-hemorrhagic lesion (placenta) in the infra umbilical region in the midline, from L3 to L5 vertebral level, just below the aortic bifurcation which appeared adherent to the sigmoid colon, jejunal loops, posterior abdominal wall and psoas muscle. Blood supply to the placenta appeared to be coming from the intestinal vessels, and branches of the uterine vessels traversing upwards (Figure 4). After discharge, the patient was followed up with monthly MRI's which showed a slow decrease in the placental size. She got her menses in 7 weeks and did not have any other complaints, such as altered bowel habits or bleeding. She was given contraceptive counselling and opted for injection depot medroxyprogesterone acetate.

\section{DISCUSSION}

Abdominal pregnancy, can be due to primary implantation in the abdomen, where intraabdominal fertilization of sperm and ovum occur, or secondary implantation from a tubal pregnancy. Though secondary implantation is more common, it may be difficult to elicit, as the defect in the fallopian tube may heal, giving normal appearance to both adnexa, as in this case. Sites of implantation include the omentum, pelvic sidewall, broad ligament, posterior cul-de-sac, abdominal organs (e.g., spleen, bowel, liver), large pelvic vessels, diaphragm and the uterine serosa.

Risk factors for abdominal pregnancy, like those of other ectopic pregnancies are tubal damage, pelvic inflammatory disease, endometriosis, assisted reproductive techniques, and multiparity. Gestational age at diagnosis varies, ranging from first trimester to full term. Patients may remain asymptomatic, or present with nausea, vomiting or changes in bowel habits due to implantation on bowel, mild vaginal bleeding, abdominal pain, or shock and death due to severe intraabdominal hemorrhage from placental separation or rupture of maternal blood vessels or viscera. ${ }^{2}$

The associated maternal mortality rate is 5 out of 1000 cases which is approximately seven times higher than tubal ectopic pregnancy and 90 times higher than intrauterine pregnancy. ${ }^{3,4}$ Our patient, however, did not have any symptoms during her pregnancy. Foetal complications include malpositions, growth restriction, foetal death and malformations, which occur in about $21 \%$ of babies, presumably due to compression of the fetus in the absence of the amniotic fluid buffer and placental insufficiency. Typical deformities include limb defects, facial and cranial asymmetry, joint abnormalities and central nervous system malformations. ${ }^{5}$
Clinical examination and imaging modalities frequently miss the diagnosis, with only about $45 \%$ of cases diagnosed during the antenatal period or misdiagnose it to be a pregnancy in a rudimentary horn or bicornuate uterus, as in our case and one reported by Dahab et al. ${ }^{6,7}$ Thus awareness about such an entity and a high index of suspicion are important for prompt diagnosis and timely intervention to prevent life-threatening complications, most common being intractable haemorrhage from the placental bed. ${ }^{8}$

Management may differ slightly based on gestational age and site of placental attachment. Basic principles include delivery of the foetus, separation of the placenta or leaving it in situ, and methods for hemorrhage control. Removal of the placenta can lead to life-threatening maternal hemorrhage, thus unless the placenta separates spontaneously, we prefer ligating the umbilical cord close to placental attachment and leaving the placenta in situ. The patient can then be monitored closely, as was done in this case. Decrease in placental volume by $83 \%$ at the end of 1 year has been noted by one study. ${ }^{9}$ Active intervention using arterial embolization or methotrexate can be instituted to hasten involution. This approach, especially when methotrexate is used has its share of complications including abscess formation, sepsis, side effects of methotrexate itself, intestinal or ureteral obstruction, fistula formation involving abdominal viscera and delayed haemorrhage. ${ }^{10}$ An alternative approach of ligating the placental blood supply and then trying to remove the placenta has been advocated by some, but the rates of blood loss and complications are higher. ${ }^{11}$

A few cases have reported the use of drugs such as Mifepristone, in varying doses, to aid in placental degeneration, as we did., ${ }^{42-16}$ Hemorrhage control measures include intraarterial occlusive catheters and embolization, autotransfusion, pelvic packing, and use of Military Anti-Shock garments. ${ }^{17}$ The use of imaging and interventional radiological techniques is critical in management, to minimize surgical interventions and post-surgical complications such as angiographic embolization or intra-arterial catheterization of feeding vessels to the placenta to decrease bleeding. ${ }^{18}$ Whatever mode of management is adopted, follow up with serial bHCG and an imaging modality (ultrasound or MRI) is essential. $^{19}$

\section{CONCLUSION}

Abdominal pregnancies, associated with a high maternal and perinatal morbidity and mortality, are diagnosed preoperatively only in $45 \%$ of cases. With the rise of assisted reproductive techniques and other risk factors, there are increasing reports of abdominal pregnancies, some reaching up to term. Thus, a high index of suspicion and improvement in diagnosis rates is the need of the hour. The cornerstones of successful management include prompt intraoperative recognition and decision regarding 
management of the placenta (we advocate leaving the placenta in situ), surgical skill and multidisciplinary approach with involvement of surgeons and interventional radiologists if required, ready access to blood products, meticulous postoperative care and close observation during the subsequent delayed reabsorption.

Funding: No funding sources

Conflict of interest: None declared

Ethical approval: Not required

\section{REFERENCES}

1. Dabiri T, Marroquin GA, Bendek B, Agamasu E, Mikhail M. Advanced extrauterine pregnancy at 33 weeks with a healthy newborn. BioMed Res Inter. 2014;2014

2. Tucker K, Bhardwaj NR, Clark E, Espey E. Delayed diagnosis and management of second trimester abdominal pregnancy. Case Reports. 2017;2017:bcr2017.

3. Gurjar R. Full-term live secondary abdominal pregnancy: a rare case report. J Obstet Gynecol India. 2018:1-4.

4. Agarwal N, Odejinmi F. Early abdominal ectopic pregnancy: challenges, update, and review of current management. Obstet Gynaecol. 2014;16:193-8.

5. Stevens CA. Malformations and deformations in abdominal pregnancy. Am J Med Genet. 1993;47:1189-95.

6. Nkusu Nunyalulendho D, Einterz EM. Advanced abdominal pregnancy: case report and review of 163 cases reported since 1946. Rural Remote Health. 2008;8:1087.

7. Dahab AA, Aburass R, Shawkat W, Babgi R, Essa O, Mujallid RH. Full-term extrauterine abdominal pregnancy: a case report. J Med Case Reports. 2011;5(1):531.

8. Worley KC, Hnat MD, Cunningham FG. Advanced extrauterine pregnancy: diagnostic and therapeutic challenges. Am J Obstet Gynecol. 2008;198(3):297e1.

9. Cetinkaya MB, Kokcu A, Alper T. Follow up of the regression of the placenta left in situ in an advanced abdominal pregnancy using the Cavalieri method. J Obstet Gynaecol Res. 2005;31(1):22-6.

10. Oneko O, Petru E, Masenga G, Ulrich D, Obure J, Zeck W. Management of the placenta in advanced abdominal pregnancies at an East african tertiary referral center. J Women's Health. 2010;19(7):136975.

11. Varma R, Mascarenhas L, James D. Successful outcome of advanced abdominal pregnancy with exclusive omental insertion. Ultrasound in Obstetrics and Gynecology: The Official J Inter Society Ultrasound Obstet Gynecol. 2003;21(2):192-4.

12. Huang K, Song L, Wang L, Gao Z, Meng Y, Lu Y. Advanced abdominal pregnancy: an increasingly challenging clinical concern for obstetricians. Inter J Clin Exper Pathol. 2014;7(9):5461.

13. Raughley MJ, Frishman GN. Local treatment of ectopic pregnancy. Semin Reprod Med. 2007;25:99115.

14. Perdu M, Camus E, Rozenberg P, Goffinet F, Chastang C, Philippe HJ, et al. Treating ectopic pregnancy with the combination of mifepristone and methotrexate: a phase II nonrandomized study. Am J Obstet Gynecol. 1998;179(3):640-3.

15. Gazvani MR, Baruah DN, Alfirevic Z, Emery SJ. Mifepristone in combination with methotrexate for the medical treatment of tubal pregnancy: a randomized, controlled trial. Human Reprod (Oxford, England). 1998;13(7):1987-90.

16. Stucki D, Buss J. The ectopic pregnancy, a diagnostic and therapeutic challenge. J Med Life. 2008;1(1):40.

17. Cardosi RJ, Nackley AC, Londono J, Hoffman MS. Embolization for advanced abdominal pregnancy with a retained placenta. A case report. The J Reprod Med. 2002;47(10):861-3.

18. Bertrand G, Le Ray C, Simard-Émond L, Dubois J, Leduc L. Imaging in the management of abdominal pregnancy: a case report and review of the literature. J Obstet Gynaecol Canada. 2009;31(1):57-62.

19. Mforteh AA, Tchakounte C, Sama CB, Eteme-Messi $\mathrm{S}$, Djiogoni W, Dohbit S, et al. Induction of labour in advanced abdominal pregnancy with fetal demise due to cord round neck: a case report of a missed diagnosis. Case Reports Obstet Gynecol. 2018:2018.

Cite this article as: Mehta NA, Fonseca MN. A rare case of abdominal pregnancy: diagnostic and therapeutic challenges. Int J Reprod Contracept Obstet Gynecol 2020;9:1288-91. 